Nervenarzt $2017 \cdot 88: 1211-1212$

https://doi.org/10.1007/s00115-017-0413-0

Online publiziert: 4. September 2017

(c) Springer Medizin Verlag GmbH 2017

CrossMark

\author{
M. Hautzinger ${ }^{1} \cdot$ M. Hüll2,3 \\ 'Fachbereich Psychologie, Klinische Psychologie und Psychotherapie, Eberhard Karls Universität, \\ Tübingen, Deutschland \\ ${ }^{2}$ Zentrum für Geriatrie und Gerontologie Freiburg - ZGGF, Universitätsklinikum Freiburg, Freiburg, \\ Deutschland \\ ${ }^{3}$ Klinik für Geronto- und Neuropsychiatrie, Zentrum für Psychiatrie Emmendingen, Emmendingen, \\ Deutschland
}

\title{
Psychotherapie mit Älteren
}

bzw. hirnorganischer Einschränkungen, Behinderungen oder Komorbiditäten, kann Psychotherapie mit entsprechender Adaptation eingesetzt werden.

Ein Fünftel der deutschen Bevölkerung hat das 65. Lebensjahr überschritten. Trotzdem ist die Literatur zur Psychotherapie mit Älteren überschaubar. Dabei ist die Auswirkung psychischer Erkrankungen im Alter auf Morbidität, Mortalität und Kosten enorm. Vielfältige Forschungsergebnisse belegen die fortbestehende Kompetenz, Plastizität, Umstellungs- und Lernfähigkeit bis ins hohe Alter. Dennoch dominiert oft noch eine pessimistische Sicht und die Abkehr vom Defizitmodell des Alterns gelingt nur langsam. Zunehmend belegen qualitativ hochwertige Studien, dass psychotherapeutische Interventionen im Alter ähnliche Wirksamkeit zeigen wie in jüngeren Jahren. Jedoch wissen viele ältere Menschen von den psychologischen, ressourcenorientierten Hilfestellungen und den wirksamen psychotherapeutischen Möglichkeiten nichts [3].

\section{》) Ältere unterscheiden sich von Jüngeren nicht grundsätzlich in der Indikation zur Psychotherapie}

Dieser Themenschwerpunkt will verdeutlichen, dass sich die Indikation zur Psychotherapie bei Älteren nicht grundsätzlich von Jüngeren unterscheidet. Hauptindikationen sind Depressionen, Angst- und Zwangsstörungen, Substanzgebrauchsstörungen, posttraumatische und akute Belastungsstörungen. Auch bei Vorliegen altersspezifischer Faktoren, wie psychosozialer, somatischer

\section{Welche psychologischen Verfahren sind Thema dieser Ausgabe?}

Bei den Psychotherapien mit Älteren dominieren die Varianten der kognitiven Verhaltenstherapie, die Problemlösetherapie, die interpersonelle Psychotherapie, die Lebensrückblicktherapie und psychodynamische Psychotherapie. Wenige davon sind speziell für ältere Patienten und deren psychische Probleme evaluiert. Die empirische Evidenz ist noch beschränkt. Gerade im Bereich der Psychotherapie mit Älteren haben therapieschulenübergreifende „Baukastenansätze“ eine lange Tradition. So werden z. B. Aspekte der interpersonellen Psychotherapie oder der Lebensrückblicktherapie oft in die Verhaltenstherapien oder psychodynamischen Therapien integriert. Ältere Menschen sind bei der Psychotherapie wie in anderen Bereichen oft anspruchsvolle und pragmatische Patienten. Der Nutzen muss für sie definierbar sein, das Vorgehen transparent und Fortschritte erkennbar. Das Misstrauen gegen theoretische Konstrukte ohne Bezug zur eigenen Lebenswelt steigt mit der Lebenserfahrung.

Die meisten Studien und die umfangreichsten klinischen Erfahrungen liegen zur kognitiven Verhaltenstherapie und dem Problemlösetraining bei der Therapie von Depressionen und chronischen bzw. posttraumatischen Belastungsstörungen vor. Dies spiegelt sich auch in den ausgewählten Beiträgen dieses Leitthemas. Die kognitive Verhaltenstherapie mit ihren verschiedenen Modulen und vielfältigen Materialien lässt sich gut in das multiprofessionelle Team einer geriatrischen Station oder in den gestuften Behandlungsplan einer ambulanten Versorgung integrieren. Das Erlernen von Problemlösetechniken bietet sich als Ansatz zur Bewältigung der vielfältigen Belastungen bei Pflegebedürftigkeit sowie zur Überwindung einer resignativ-depressiven Symptomatik an. In der Behandlung posttraumatischer Belastungsstörungen verspricht die bewährte kognitive Verhaltenstherapie auch bei älteren Patienten Validität und Effizienz.

\section{Ein Leitthema ist nicht genug}

Mit den hier vorgestellten Anwendungsbereichen ist das Feld der Psychotherapie mit Älteren längst nicht abgedeckt. Es gibt sowohl auf nationaler als auch auf internationaler Ebene Therapiestudien und Programme (Manuale) zum Nutzen der Psychotherapie, z. B. nach Schlaganfällen $[1,6]$, bei Diabetes [5], bei Morbus Parkinson [2, 8], bei Sturzängsten nach Frakturen [4], bei Demenz [9] oder bei Makuladegeneration und Sehkraftverlusten [7].

\section{Botschaft dieses Leitthemas}

Ältere Menschen sind heutzutage im Schnitt in ihrer körperlichen Leistungsfähigkeit 10 Jahre jünger als die vorherige Generation. Gleichzeitig steigen die Ansprüche und Erwartungen an Selbstständigkeit und Teilhabe. Niemals zuvor haben so viele ältere Menschen 
nach dem 65. Lebensjahr noch bezahlte Tätigkeiten ausgeführt, Enkelkinder betreut oder einen unabhängigen Haushalt geführt. Diese Selbstständigkeit und Teilhabe gilt es auch mit psychotherapeutischen Mitteln zu erhalten, sowohl aus der Perspektive der Gesundheit des Einzelnen als auch aus der Perspektive der Gesellschaft, die mit ihren rein pflegerisch-versorgenden Angeboten schon jetzt an ihre Grenzen stößt. Es gibt keinen Grund, älteren Patienten diese wirksame Hilfe vorzuenthalten.<smiles>CC#CC</smiles>

M. Hautzinger

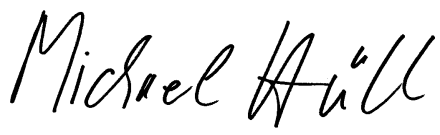

M. Hüll

\section{Korrespondenzadresse}

Prof. Dr. M. Hautzinger

Fachbereich Psychologie, Klinische Psychologie und Psychotherapie, Eberhard Karls Universität Schleichstr. 4, 72076 Tübingen, Deutschland hautzinger@uni-tuebingen.de

Interessenkonflikt. M. Hautzinger und M. Hüll geben an, dass kein Interessenkonflikt besteht.

\section{Literatur}

1. Bauder H, Taub E, Miltner W (2001) Behandlung motorischer Störungen nach Schlaganfall. Hogrefe, Göttingen

2. Dobkin RD, Menza M, Allen LA et al (2011) CBT in Parkinson disease. A randomized, controlled trial. Am JPsychiatry 168:1066-1074

3. Fellgiebel A, Hautzinger M (2017) Altersdepression. Ein interdisziplinäres Handbuch. Springer, Heidelberg

4. Kampe K, Kohler M, Albrecht D, Becker C, Hautzinger M, Lindemann U, Pfeiffer K (2017) Hip and pelvic fracture patients with fear of falling. Development and description of the Step-by-Step treatment protocol. Clin Rehabil. https://doi.org/ 10.1177/0269215517691584

5. Petrak F (2016) Ältere Menschen mit Diabetes ein kognitiv-verhaltenstherapeutisches Manual. Springer, Heidelberg

6. Pfeiffer K (2017) Depression nach Schlaganfall (Kap. 20). In: Fellgiebel A, Hautzinger M (Hrsg) Altersdepression. Ein interdisziplinäres Handbuch. Springer, Heidelberg

7. Rovner BW, Casten RJ, Hegel MT, Leiby BE, Tasman WS (2007) Preventing depression in age- related macular degeneration. Arch Gen Psychiatry 64:886-892

8. Strehl U, Birbaumer N (1996) Verhaltensmedizinische Intervention bei Morbus Parkinson. Beltz, Weinheim

9. Werheid K, Thöne-Otto A (2010) Alzheimer Krankheit. Ein neuropsychologisch-verhaltenstherapeutisches Manual. Beltz, Weinheim

\section{T. Poehlke, W. Heinz, H. Stöver \\ Drogenabhängigkeit und Substitution}

\section{Ein Glossar von A-Z}

Berlin Heidelberg: Springer-Verlag 2016, 4. Aufl., (ISBN: 978-3-66249848-4), Softcover 19,99 EUR

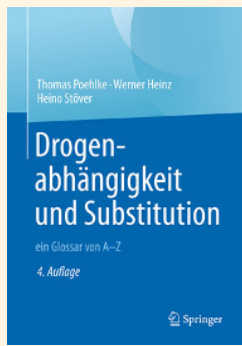

Es handelt sich um ein kompaktes Büchlein, in dem die Autoren zahlreiche Themenartikel zusammengetragen haben, die mit der Substi-

tutionsbehandlung Opiatabhängiger zu tun haben. Der Glossarcharakter führt zu voneinander unabhängigen Artikeln, die thematische Überschneidung und damit zusammenhängende Redundanzen halten sich erfreulich gering. Die Beschreibungen der Themenbereiche sind immer dann besonders sicher, wenn sich die Autoren mit den Grundlagen und der Praxis der Substitutionsbehandlung auseinandersetzen. Die der Substitution in Deutschland zugrunde liegenden Gesetze, Verordnungen und Rahmenbedingungen nehmen einen großen Raum ein und werden entsprechend ihrer Bedeutung für den einzelnen Behandler verständlich erläutert. Dem substituierenden Psychiater mögen gelegentlich Schwerpunktsetzungen auf dem eigenem Fachgebiet fremd erscheinen. Als Nachschlagewerk zu Fragen der Substitution ist es aber gut geeignet. Dank seiner Kompaktheit eignet es sich gut, in einem Stück gelesen zu werden, um einen schnellen Überblick über die Substitutionsbehandlung zu gewinnen.

Die Betäubungsmittelverschreibungsverordnung, die die Substitutionsbehandlung in Deutschland gesetzlich regelt, und die Richtlinien der Bundesärztekammer zur Substitution wurden gerade geändert. Die Auswirkungen auf die Behandlungspraxis sind bereits abzusehen, so dass eine Neuauflage des Bandes sicher bald folgen wird. Fazit: Gut geeignet, um schnell eine Übersicht über die Substitutionsbehandlung und angrenzende Themen zu gewinnen.

\section{Vollmert (Mannheim)}

